\title{
Opportunities to Improve COVID-19 Dashboard Designs for the Public
}

\author{
Helen MONKMAN a,1, Susan Z. MARTIN a, Simon MINSHALL a , Andre W. \\ KUSHNIRUK ${ }^{\mathrm{a}}$, and Blake J. LESSELROTH ${ }^{\mathrm{a}, \mathrm{b}}$ \\ ${ }^{a}$ School of Health Information Science, University of Victoria, Victoria, B.C., Canada \\ ${ }^{\mathrm{b}}$ University of Oklahoma School of Community Medicine, Tulsa, Oklahoma, USA
}

\begin{abstract}
Many organizations created COVID-19 dashboards to communicate epidemiologic statistics or community health capabilities with the public. In this paper we used dashboard heuristics to identify common violations observed in COVID-19 dashboards targeted to citizens. Many of the faults we identified likely stem from failing to include users in the design of these dashboards. We urge health information dashboard designers to implement design principles and test dashboards with representative users to ensure that their tools are satisfying user needs.
\end{abstract}

Keywords. Consumer health informatics, dashboards, human-centred design

\section{Introduction}

The coronavirus or COVID-19 pandemic was an existential threat that exposed vulnerabilities in our public health response systems. People and organizations struggled to understand and adapt to the rapidly changing clinical climate. Health organizations, public health officials, and other stakeholders recognized an urgent need to broadcast information about outbreak status, transmission rates, and vaccinations. Many developed dashboards in an attempt to communicate this information with the public. It is unclear, however, whether dashboards (1) are commonly adopted by users; (2) efficiently communicate relevant trends and patterns; or (3) satisfy users' information needs.

A dashboard is "a visual display of data used to monitor conditions and/or facilitate understanding" [1]. COVID dashboards displays and features vary across products from tables of statistics with icons (e.g., [2]) to static charts and maps, to interactive visualizations. COVID dashboards also vary by scope, data domain, or span. For example, some focus on micro-systems (e.g., hospital or city) whereas others focus on macro-systems (e.g., country or continent).

Dashboards proliferated during the pandemic, but as the old adage goes, "just because you can, doesn't mean you should". Organizations often fail to communicate key information, rarely revise their dashboards designs after release, and even decommission their dashboards despite ongoing need (e.g., [3]). Using a set of published dashboard heuristics, we examined COVID dashboards to understand their shortcomings and recommend what designers can do to improve their effectiveness.

${ }^{1}$ Helen Monkman, School of Health Information Science, University of Victoria, Victoria, British Columbia, Canada; E-mail: monkman@uvic.ca 


\section{Methods}

Our team of user experience experts assessed COVID dashboards holistically using Dowding and Merrill's 10 Heuristics for dashboard visualizations [4] (Table 1). These heuristics are based on a subset of Nielsen's heuristics [5] and several heuristics specific to information visualization [6]. Two inspectors (HM, SZM) independently examined a sample of 5 dashboards available to Canadian citizens (e.g., regional, provincial, international) to identify heuristic violations. Rather than critique a single dashboard, the purpose of this evaluation was to summarize common usability violations across products and organized by heuristic. Hence, each inspector documented violations and generated opportunities for improvement separately. Due to space limitations, we jointly selected exemplars from the independent evaluations for the results section.

Table 1. Dowding and Merrill's 10 Heuristics for Dashboard Visualizations [4] (p. 513-514).

\begin{tabular}{|c|c|}
\hline Heuristic & Description \\
\hline $\begin{array}{l}\text { 1. Visibility of System } \\
\text { Status }\end{array}$ & $\begin{array}{l}\text { "The system should always keep user informed about what is going on } \\
\text { through appropriate feedback within reasonable time" }\end{array}$ \\
\hline $\begin{array}{l}\text { 2. Match Between the } \\
\text { System and the Real } \\
\text { World }\end{array}$ & $\begin{array}{l}\text { "The system should speak the user's language, with words, phrases, and } \\
\text { concepts familiar to the user, rather than system oriented terms. Follow real- } \\
\text { world conventions, making information appear in a natural and logical order" }\end{array}$ \\
\hline $\begin{array}{l}\text { 3. User Control and } \\
\text { Freedom }\end{array}$ & $\begin{array}{l}\text { "Users should be free to select and sequence tasks (when appropriate), rather } \\
\text { than having the system do this for them. Users will need a clearly marked } \\
\text { "emergency exit" to leave the unwanted state without having to go through an } \\
\text { extended dialogue. Users should make their own decisions regarding the costs } \\
\text { of exiting current work" }\end{array}$ \\
\hline $\begin{array}{l}\text { 4. Consistency and } \\
\text { Standards }\end{array}$ & $\begin{array}{l}\text { "Users should not have to wonder whether different words, situations, or } \\
\text { actions mean the same thing." }\end{array}$ \\
\hline $\begin{array}{l}\text { 5. Recognition Rather } \\
\text { than Recall }\end{array}$ & $\begin{array}{l}\text { "Make objects, actions and options visible. The user should not have to } \\
\text { remember information from one part of the dialogue to another. Instructions for } \\
\text { the use of the system should be visible or easily retrievable whenever } \\
\text { appropriate" }\end{array}$ \\
\hline $\begin{array}{l}\text { 6. Flexibility and } \\
\text { Efficiency of Use }\end{array}$ & $\begin{array}{l}\text { "The system should offer users several options when it comes to finding } \\
\text { content. Users should be able to achieve their goals in an efficient manner" }\end{array}$ \\
\hline 7. Aesthetics and & "Dialogues should not contain information which is irrelevant or rarely needed. \\
\hline Minimalist & Every extra unit of information in a dialogue competes with the relevant units \\
\hline $\begin{array}{l}\text { Design/Remove the } \\
\text { Extraneous (Ink) }\end{array}$ & $\begin{array}{l}\text { of information and diminishes their relative visibility. Present the largest } \\
\text { amount of data with the least amount of ink" }\end{array}$ \\
\hline 8. Spatial & "Relates to the overall layout of a visual representation and refers to how easy \\
\hline Organization & $\begin{array}{l}\text { it is to locate an information element in the display and the distribution of } \\
\text { elements in representations" [3] (p. 514) }\end{array}$ \\
\hline 9. Information Coding & "Refers to the use of symbols or representations to aid perception" \\
\hline 10. Orientation & $\begin{array}{l}\text { "Provision of support for the user and help to orientate them in the } \\
\text { visualization" }\end{array}$ \\
\hline
\end{tabular}

\section{Results}

\subsection{Visibility of System Status}

Generally, COVID-19 dashboards effectively communicated system status visually (i.e., displayed throbbers to indicate the data was loading). Some dashboards initially loaded slowly but responded more quickly with subsequent tasks. For some dashboards the currency of the data was not obvious. That is, data labels said "new" without qualifying the date of collection or reporting). 


\subsection{Match Between the System and the Real World}

Many dashboards included technical jargon, but neglected key information needed for strategic decisions. For example, one dashboard had tabs arbitrarily labelled (e.g., "Admin0"), that when selected, allowed users to drill down in the data [7]. Another dashboard listed some of the total cases as "epi-linked", but did not include a definition for this term [8]. Many displays included raw data (the numerator) without the population size (the denominator), thus preventing any comparison between regions. Finally, rather than summarizing measures (e.g., 182.8 million), dashboards often included numbers with an excessive level of precision (e.g., 182,825,864). Larger numbers are harder to compare [9]. Therefore, dashboard designers should strive to summarize large numbers.

\subsection{User Control and Freedom}

The dashboards we reviewed rarely included "accelerators", keyboard shortcuts, or other affordances for power users (e.g., control $+z$, browser back button). Some dashboards included "home" buttons that only worked on a portion of the dashboard (typically a map). Users typically had no way to restore the dashboard to the default view (e.g., [8]).

\subsection{Consistency and Standards}

There was little consistency across dashboard designs, and we identified missed opportunities for countries to standardize displays for their citizens. For example, the Canadian provinces and territories could have created a standard design with the flexibility to provide regional context (e.g., locally relevant resources and population specifics). A standard design with configurable controls could enable users to easily drill down in the data for regional trends, such as epidemiologic patterns within cities (e.g., [10]). Also, within the same dashboard, two similar looking figures had a note below to indicating that the scales on the Y axis differed, which could easily be overlooked [8].

\subsection{Recognition Rather than Recall}

Many dashboards exceeded the display capacity of a single screen, forcing users to scroll or switch between screens (e.g., [11]). If users cannot see all data on a single screen, they must recall information from their working memory to compare and analyze values. Similarly, if filter settings, column headers, and labels are not on-screen, users may struggle to integrate data or recognize when they are looking at a subset of the data. One dashboard used a date legend (i.e., M/D/YYYY) [7], but rather than requiring users to recall the date format, the date display could be disambiguated (e.g., 3-Jul-2021, 3 July 2021) to facilitate comprehensibility [12] and free up screen real estate.

\subsection{Flexibility and Efficiency of use}

Dashboards designers rarely included features that predicted user needs, added value, or connected users with related resources. For example, designers could have re- directed online traffic to local testing locations or symptom screening questionnaires. In many cases it appeared that the dashboards were islands unto themselves, lacking connections to helpful information about their source data or validation methods. 


\subsection{Aesthetics and Minimalist Design/Remove the Extraneous (Ink)}

Many dashboards included unnecessary or excessive words, graphics, and visualizations that distracted from the main message. For example, one dashboard used valuable screen real estate for unintuitive or minimally valuable icons (e.g., [8]). Some displays extended beyond a single screen leaving information "below the fold" [11]. Additionally, designs tended to display everything at once, rather than using a progressive disclosure strategy.

\subsection{Spatial Organization}

Dashboards ranged from simple and uncluttered to busy and disorganized. Some dashboards allowed users to modify the display such as column size (e.g., [8]). Unfortunately, these options offered little value, and in some cases, could not be easily undone. Dashboards with maps occasionally included additional information as a transient "mouseover" note. However, users either had no way to capture the information in a snapshot, or the message altered the underlying map.

\subsection{Information Coding}

Dashboards rarely indicated when officials implemented new public health protocols or lifted restrictions, making it impossible to ascertain the public health impact of policy interventions. When reporting daily statistics, dashboards rarely included graphic depictions of trends (e.g., [11]) such as arrows to indicate directionality.

Colour was another common problem with COVID-19 dashboards. Many dashboards used red to convey the number of COVID-19 cases and/or had black backgrounds. In terms of colour semantics, in Western cultures red is often associated with danger [13] and black with grief. Additionally, some paired red with green, creating a problem for people with colour-blindness [14]. Finally, some developers used the same colour to encode several different meanings simultaneously.

\subsection{Orientation}

Our experts generally agreed that most of landing pages we reviewed helped orient users by including clear titles and maps. However, when users applied filters, this compromised orientation. In one dashboard example, when a geographic filter was applied, it applied the filter to each section, rather than keeping the geographic section highlighted and in view [11].

\section{Discussion and Conclusion}

There has been rapid growth in COVID-19 dashboards designed for the spectrum of health care stakeholders (e.g., epidemiologists, public). However, our UX experts agreed that many dashboards violated dashboard design heuristics indicating frequent usability issues that may have created challenges for citizens. Thus, many dashboards likely performed as suboptimal communication tools amidst this global pandemic.

It is even more problematic when we consider that the target user of these dashboards is often the healthcare consumer. Over half of Canadians have inadequate 
numeracy skills [15] and even more struggle with low health literacy [16]. Yet, while many developers ostensibly created dashboards to inform and empower the public, few leveraged lessons learned from usability research. The accessibility issues and jargon instead risk potentiating an existing health literacy gap. Based on the shortcomings observed, it is doubtful dashboard designers used a human-centred design approach [17]. Therefore, many of these dashboards may have generated more confusion than clarity in an already sensitive, stressful, and uncertain time. In conclusion, we believe that dashboards could be valuable components of public health initiatives and communicate crucial information with their users. However, to achieve this and empower health care stakeholders, it is vital that developers adopt a human-centred approach. Our public health campaigns as well as our design and communication strategies should be evidence based and engage users throughout the development lifecycle.

\section{References}

[1] S. Wexler, J. Shaffer, and A. Cotgreave, The big book of dashboards: visualizing your data using realworld business scenarios, Wiley, Hoboken, New Jersey, 2017.

[2] Province of Manitoba | Cases and Risk of COVID-19 in Manitoba, Prov. Manit. (n.d.). https://www.gov.mb.ca/covid19/updates/cases.html (accessed July 4, 2021).

[3] K. Local4, State COVID-19 Dashboard concludes as emergency ends, Https://Www.Knopnews2.Com. (n.d.). https://www.knopnews2.com/2021/06/30/state-covid-19-dashboard-concludes-emergency-ends/ (accessed July 4, 2021).

[4] D. Dowding, and J.A. Merrill, The Development of Heuristics for Evaluation of Dashboard Visualizations, Appl. Clin. Inform. 9 (2018) 511-518. doi:10.1055/s-0038-1666842.

[5] J. Nielsen, 10 Usability Heuristics for User Interface Design, Nielsen Norman Group. (n.d.). https://www.nngroup.com/articles/ten-usability-heuristics/ (accessed January 31, 2021).

[6] C. Forsell, and J. Johansson, An heuristic set for evaluation in information visualization, in: Proc. Int. Conf. Adv. Vis. Interfaces - AVI 10, ACM Press, Roma, Italy, 2010: p. 199. doi:10.1145/1842993.1843029.

[7] COVID-19 Map, Johns Hopkins Coronavirus Resour. Cent. (n.d.). https://coronavirus.jhu.edu/map.html (accessed July 4, 2021).

[8] B.C.

COVID-19

Dashboard,

(n.d.). https://experience.arcgis.com/experience/a6f23959a8b14bfa989e3cda29297ded (accessed July 4, 2021).

[9] R.S. Moyer, and T.K. Landauer, Time required for Judgements of Numerical Inequality, Nature. 215 (1967) 1519-1520. doi:10.1038/2151519a0.

[10] COVID-19: Neighbourhood Maps, City Tor. (2021). https:/www.toronto.ca/home/covid-19/covid-19latest-city-of-toronto-news/covid-19-pandemic-data/covid-19-neighbourhood-maps-data/ (accessed July 4, 2021).

[11] Saskatchewan's Dashboard - Health and Wellness, Gov. Sask. (n.d.). https://dashboard.saskatchewan.ca/health-wellness (accessed July 4, 2021).

[12] Clinical Application and Patient Safety Project, NHS CUI Design Guide Workstream: Design Guide Entry - Date Display, Health \& Social Care Information Centre (HSCIC), n.d.

[13] C. Ware, Information visualization: perception for design, Fourth, Elsevier, Inc, Philadelphia, 2020.

[14] Color vision deficiency, (n.d.). https://www.aoa.org/healthy-eyes/eye-and-vision-conditions/colorvision-deficiency?sso=y (accessed July 3, 2021).

[15] The Conference Board of Canada, Adults With Inadequate Numeracy Skills, (n.d.). https://www.conferenceboard.ca/hcp/provincial/education/adlt-lownum.aspx (accessed July 4, 2021).

[16] Canadian Council on Learning, Health literacy in Canada: A healthy Understanding, 2008.

[17] ISO 9241-210:2019(en), Ergonomics of human-system interaction — Part 210: Human-centred design for interactive systems, (n.d.). https://www.iso.org/obp/ui/\#iso:std:iso:9241:-210:ed-2:v1:en (accessed July 4, 2021). 